\title{
Foreword for the Special Issue "The Economics of Digital Business Models"
}

\author{
ERIC BROUSSEAU ${ }^{1}$ \\ EconomiX, Université Paris X and IUF, France \\ THIERRY PENARD \\ CREM, Université Rennes 1 and Marsouin, France
}

Over the past decade, the distribution/growth of information technology (IT) has transformed the organization of economic activities. IT has enhanced economic agents' capabilities to collect and process information, to coordinate and manage transactions. Although IT threatens the activities of traditional intermediaries, it also creates new opportunities and needs for intermediation. This special issue aims to define these new forms of intermediation and understand their underlying business models. This issue is composed of a selection of papers presented at the workshop, "The economics of $e$ intermediation: What is specific in digital business models?” held in Paris (June 2005).

This special issue proposes theoretical lenses for contrasting and characterizing new digital business models (DBM). A DBM is a three-dimensional model that combines elements of matching, assembling and knowledge management. The selected papers provide in-depth and complementary views on these three dimensions.

The first dimension refers to the economics of matching and focuses on transaction costs and network externalities. Digital networks are generally understood to be market places where supply meets demand. The performance of transactions between "providers" and "consumers" (of information and services) requires dedicated resources to solve a set of transactional difficulties (matching demand and supply, secure transactions, risk management, etc.). Taking this approach, DBMs can be analyzed through their capacity to organize transactions and stimulate network externalities between the two sides of the "market".

The second dimension refers to the economics of assembling and focuses on assembling costs and users' preferences for alternative composite goods. The role of assemblers is to combine content and functionalities to supply "packages" that fit the heterogeneous needs of users. DBMs refer to the alternative ways of settling trade-offs in terms of scope of assembling (and correlated degrees of integration between components) and strategies to extract value from users.

The third dimension refers to the economics of knowledge management and examines how IT can stimulate innovation by providing tools for managing and sharing information

\footnotetext{
${ }^{1}$ The guest editors of this volume would like to thank the Centre National de la Recherche Scientifique (CNRS) for backing their research with the "Information Society” Program. We also received financial support from France Télécom Research and Development, the CDC Foundation for Research, the European Union (DG Research as part of the "Ref-Gov" Integrated project) and Marsouin (Brittany region). We would like to warmly thank all these sponsors. The usual caveats apply.
} 
and knowledge generated by digital goods users, who become (passive or active) contributors. Digital business models can be defined by their different capabilities to efficiently accumulate and create knowledge. Differences between models result from individual incentives to share information with others, and from the ability of each individual innovator to access and retrieve the relevant information and knowledge.

Three key points must be emphasized here. First, we speak of dimensions because we view the design of a business model by an entrepreneur or a community as a positioning within this three-dimension space. Indeed, they have to choose a way to organize transactions, to assemble functionalities and to manage knowledge when they want to produce and distribute digital goods. Each of these choices can be (and often is) analyzed separately, but any DBM corresponds to a set of choice in each of these dimensions. Second, we consider these dimensions as independent. The choices made in terms of how transactions are organized are independent of those made regarding knowledge management. This is even true when we speak of "intermediation". The matching of demand and supply can be centralized by an intermediary, but knowledge management is decentralized; and vice versa. Studying some business models using one of these dimensions for reference only is therefore useful for understanding the economics of many business models. Third, it is, however, vital to take into account the general economics of each of the possible business models by taking the three dimensions highlighted in this framework into consideration together. Monopolies being sustainable in a digital economy, a dominant player tends to emerge in each niche. These players correspond to each of the possible combinations of available solutions for providing the service, in accordance with the three dimensions described above. Competition is therefore between business models (characterized by contrasted economics), rather than between players adopting identical business models. A good example of this can be seen in the competition between Linux and Microsoft, between Amazon and the shopbots, for instance.

The trade-offs (and their combinations) for these three dimensions are summed up in the first contribution to this issue by ourselves (Eric Brousseau and Thierry Pénard). The other papers study the details of these trade-offs by exploring the economics of alternative models of matching, assembling and knowledge management, respectively.

The papers by Andrei Hagiu and by Michael Arnold and Thierry Pénard refer to the economics of matching. Andrei Hagiu analyzes the tradeoffs between two polar modes of intermediation: the "merchant" mode, in which the intermediary buys from sellers and resells to buyers; and the "two-sided platform" mode, according to which the intermediary enables affiliated sellers to sell directly to affiliated buyers. The choice between these two business models depends upon the extent of indirect network externalities (between buyer and sellers), and the degree of substituatibility/complementarity between sellers' products. Michael Arnold and Thierry Pénard examine how infomediaries like Autobytel, which offers online referral services for the automobile market, can affect price setting and market competition: that is, do they hinder or intensify price competition? The impact of referral intermediaries depends mainly on the degree of heterogeneity among consumers' preferences or bargaining abilities, but also on the characteristics of the product and reach of the infomediaries.

The two next papers (Marc Bourreau, Pinar Dogan and Matthieu Manant; Nicolas Curien and François Moreau) focus on the economics of assembling. Nicolas Curien and François Moreau examine the assembling strategies between content and access industries. Their findings reveal that given the positive consumption externalities between content 
categories (music, video, etc.) and access networks or technologies (fixed broadband, 3G mobile, digital players, etc.), both industries have an interest in linking their business models. Because digital piracy benefits access providers (but is detrimental to content providers), a "win-win" game is only possible if access providers participate in content production, either through subsidies or joint-production. Marc Bourreau et al focus on the modular design of digital goods and its impact on innovation. Modular product design, compared to integrated (or integral) design, facilitates innovation since only parts of the product have to be "reinvented". At the same time, it provides for considerably more complex innovation strategies, since innovations must be compatible with the existing base (and parallel innovations), resulting in coopetition (competition together with cooperation) between firms,

The last three papers (Ramayya Krishnan, Michael D. Smith, Zhulei Tang and Rahul Telang; Fang Fang, Maxwell Stinchcombe and Andrew Whinston; Alex Gaudeul) deal with the economics of knowledge management and, more generally, with the economics of the common production of public or club goods. This is the key dimension in the contribution by Ramayya Krishnan et al, who review the economic characteristics of P2P networks. They outline the implications of a peer-based production of collective resources on potential uses of P2P networks in new business models of content distribution. Peer-topeer (P2P) services allow users to share networked resources, notably bandwidth and content, from the edges of the network. These services have become popular because of file sharing; particularly the sharing of unlicensed copyrighted files. However, content owners are increasingly exploring the ability of peer-to-peer networks to accommodate legitimate content distribution and promotion. More generally, this paper can be read as a contribution to a better understanding of how specific rules for sharing capacities can lead to an optimization of the use/production of digital and information resources. Fang Fang et al propose a business model of betting mechanisms and crowd sourcing for eliciting costly, dispersed information of differing quality, which is important on the Internet. Their aim is to propose an efficient way of encouraging contribution and managing quality when the production of information (here forecasted) is based on contributions from non-ex ante sorted contributors. Their paper helps better understand how crowd sourcing can be managed to attain efficiency and quality. Finally, Alex Gaudeul examines the economics of open source software by pointing out how the design of OSS cannot escape competitive consideration. OSS developers are seen as entrepreneurs needing to develop sustainable business models by considering competition, and therefore users' needs. His approach is based on a case study of $\mathrm{T}_{\mathrm{E}} \mathrm{X}$, the open source typesetting program. This software competes with a variety of open source and proprietary alternatives. In this case study, Alex Gaudeul argues that open source software constitutes a viable business model as long as developers derive direct and indirect network externalities from usage of their software by others, and take the needs of non-developers into account to make their software more attractive to a broader audience, and more competitive with proprietary alternatives. 